\title{
Natural Besnoitia besnoiti infections in cattle: hematological alterations and changes in serum chemistry and enzyme activities
}

Martin C Langenmayer ${ }^{1 *}$, Julia C Scharr ${ }^{2}$, Carola Sauter-Louis ${ }^{3}$, Gereon Schares ${ }^{4}$ and Nicole S Gollnick ${ }^{3}$

\begin{abstract}
Background: The emerging disease bovine besnoitiosis is caused by the apicomplexan parasite Besnoitia besnoiti. Clinical signs of acute besnoitiosis are pyrexia, anorexia and subcutaneous edema. In subacute and chronic besnoitiosis parasitic cysts arise in a variety of tissues and affected cattle display skin lesions and weight loss. In all stages of bovine besnoitiosis, lesions can be found in many organ systems and therefore presumably alter a variety of laboratory parameters. In this study, the impact of naturally acquired acute, subacute and chronic bovine besnoitiosis on hematologic parameters, serum chemistry, and enzyme activities was investigated. Laboratory parameters of two Simmental heifers and two Limousin cows were monitored during acute, subacute and chronic besnoitiosis and in another Simmental heifer during subclinical besnoitiosis. To determine aberrations of laboratory parameters, values were compared with reference ranges obtained from B. besnoiti negative Simmentals (224 samples of nine animals) and Limousins (41 animals). Further, laboratory parameters of $B$. besnoiti seropositive Limousin cows (54 animals; 32 of these showing clinical signs) and healthy $B$. besnoiti seronegative Limousin cows (41 animals) were compared.

Results: During acute and subacute besnoitiosis, a reduction of leukocyte and erythrocyte concentrations, hematocrit, serum albumin, urea, magnesium, and calcium concentrations were observed. Serum total protein, globulin, total bilirubin and creatinine concentrations were increased and aspartate transaminase (AST) and creatine kinase (CK) activities were elevated. In chronic besnoitiosis, erythrocyte parameters were statistically significantly lower, and total protein and globulin concentrations were significantly higher in B. besnoiti seropositive compared with $B$. besnoiti seronegative Limousin cows.

Conclusions: In this study, altered laboratory parameters during the course of naturally acquired acute, subacute and chronic bovine besnoitiosis are described for the first time. Only a few animals were examined in acute and subacute besnoitiosis, however the alterations of laboratory parameters during these stages reflected i) the acute inflammatory state (e.g. high levels of serum globulin fractions), ii) clinical findings such as disturbed condition (e.g. bilirubin concentrations), and iii) lesions such as muscle necroses described in the literature (e.g. AST or CK activities). Chronic besnoitiosis led to typical alterations of chronic inflammatory diseases like hyper-(gamma)globulinemia or reduced erythrocyte concentrations.
\end{abstract}

Keywords: Bovine besnoitiosis, Besnoitia besnoiti, Hematological and biochemical parameters, Cattle

\footnotetext{
* Correspondence: langenmayer@patho.vetmed.uni-muenchen.de

${ }^{1}$ Institute of Veterinary Pathology at the Centre for Clinical Veterinary

Medicine, Ludwig-Maximilians-Universitaet Muenchen, Munich, Germany

Full list of author information is available at the end of the article
} 


\section{Background}

Besnoitia besnoiti, a cyst-forming apicomplexan protozoon, is the causative agent of bovine besnoitiosis. Bovine besnoitiosis has spread within Europe in the past few years, with latest outbreaks in Hungary [1], Switzerland [2], Italy [3], and Germany [4]. In 2010, the disease has been classified as "emerging disease" by the European Food Safety Authority [5]. Clinical signs of infected cattle in the early stage of the disease include fever, edema, enlarged lymph nodes, anorexia, weight loss and, in bulls, swollen and painful testes [6-8]. In the chronic stage, parasitic cysts arise in various organs, including skin, vascular walls, scleral conjunctivae, and other non-intestinal mucous membranes [9-11].

Clinical signs, morphological changes, pathological lesions, parasitological examinations and diagnosis of bovine besnoitiosis have been in the focus of researchers $[6,9,12-16]$. However, the effects of the parasitic infection on laboratory parameters during acute and chronic stages of the disease were not investigated.

$B$. caprae, a parasite very closely related to B. besnoiti $[17,18]$, causes similar clinical signs in goats $[19,20]$ and data on altered laboratory parameters in caprine besnoitiosis are already available. In naturally acquired caprine besnoitiosis in Iran, alterations of various hematological and biochemical parameters as well as enzyme activities have been observed [20,21]. However, similar examinations regarding bovine besnoitiosis are missing, and metabolic disturbances caused by tachyzoites and bradyzoites are unknown.

We hypothesized that pathological alterations during the acute stage like hemorrhages, necroses or degenerative lesions in different organs, for example muscle and liver cells, and activation of lymphatic tissues [15] lead to alterations of laboratory parameters connected to those lesions. Hemorrhages may affect erythrocyte parameters, muscle and liver necroses may lead to an increase in organ specific enzyme activities, and alterations of lymphatic tissues or multifocal inflammation may affect leukocyte parameters.

In the chronic stage, we hypothesized that emaciation and the huge number of tissue cysts partially surrounded by chronic granulomatous inflammation in different organs $[4,9,22]$ may negatively affect serum albumin concentrations (emaciation) or elevate serum globulin concentrations (chronic inflammation).

The objective of the present study was to monitor laboratory parameters during acute, subacute and chronic naturally acquired bovine besnoitiosis and to connect those findings to already obtained clinical and serological findings in the same animals [13,23,24]. In addition, we aimed to compare these findings with laboratory parameters of $B$. besnoiti seronegative cattle and altered parameters of a larger group of chronically infected seropositive Limousin cattle from a German cow-calf-operation (Herd-BbGER1) [13,23].

\section{Methods}

\section{Animals}

Chronology of acute, subacute, and chronic bovine besnoitiosis monitoring infected Simmental and Limousin cattle

Animals were part of a longitudinal study focusing on the different stages and disease progression of naturally acquired bovine besnoitiosis [13,23]. Permission for this study was granted by the responsible authorities (Animal ethics committee, regional government of Upper Bavaria, TV Az. 55.2-54-2531-83-09). The study consisted of a 12week cohabitation period (August 18, 2009, until November 9, 2009). Five healthy German Simmental heifers (Study Animal [SA] 3, SA 4, SA 6, SA 8, and SA 9) and one healthy German Simmental bull (SA 1) were kept on a pasture with three chronically infected and B. besnoiti seropositive Limousin cows. Six healthy Simmental heifers (SA 2, SA 5, SA 7, SA 10, SA 11, and SA 12) between 11 and 20 months of age were kept on a paddock $20 \mathrm{~m}$ away as a control group [23]. Prior to the trial, the Simmentals tested negative for B. besnoiti and Neospora caninum antibodies in immunoblots and IFAT [24].

On trial day (td) 3 and td 51, two Limousin cows (SA 20 and SA 22) of Herd-BbGER1 suspected to be in the acute stage of bovine besnoitiosis, were added to the pasture. Infection of SA 4, SA 6, SA 20, and SA 22 was confirmed clinically, histologically, and serologically. In the case of SA 8, infection was confirmed serologically (Table 1) [23]. Although SA 22 had already seroconverted on the day of admission, this day was regarded as day of seroconversion to make inter-animal comparison possible for data analyses.

The acute stage was classified according to clinical criteria. Animals had to show fever (body temperature > $39.0^{\circ} \mathrm{C}$ ) or at least two of the following clinical signs/

Table 1 Overview of $B$. besnoiti affected Simmental (SA 4, SA 6, and SA 8) and Limousin (SA 20 and SA 22) cattle

\begin{tabular}{llllll}
\hline Animal ID & SA 4 & SA 6 & SA 8 & SA 20 & SA 22 \\
Breed & S & S & S & L & L \\
\hline Age (months) & 20 & 19 & 13 & 53 & 49 \\
Pregnancy status & $\mathrm{np}$ & $\mathrm{np}$ & $\mathrm{np}$ & $\mathrm{p}$ & $\mathrm{p}$ \\
Study entry (td) & 1 & 1 & 1 & 3 & 51 \\
Acute stage: start (td) & 36 & 29 & - & unknown & unknown \\
Acute stage: end (td) & 47 & 41 & - & 7 & 51 \\
Seroconversion (td) & 45 & 35 & 73 & 4 & - \\
Chronic stage: start (td) & 64 & 67 & - & 27 & 63 \\
Course of disease & mild & mild & subclinical & severe & severe \\
\hline
\end{tabular}

$\mathrm{SA}=$ Study animal, $\mathrm{S}=$ Simmental, $\mathrm{L}=$ Limousin, $\mathrm{np}=$ non pregnant, $\mathrm{p}=$ pregnant, $\mathrm{td}=$ trial day. 
diagnoses of acute besnoitiosis: depression, conjunctivitis, subcutaneous edema, lymphadenitis, lameness. As soon as cysts were clinically observed in the scleral conjunctivae, the term 'chronic stage' was used (Table 1). The subacute stage was defined as stage between the end of the acute and the beginning of the chronic stage.

Blood samples were collected twice a week during the whole trial period and daily for 21 days after animals showed signs of acute besnoitiosis. On day 225, SA 4, SA 6, SA 8, SA 20, and SA 22 were bled during a routine herd health status examination.

In total, 224 samples collected from the control heifers and SA 1, SA 3, and SA 9 were used to determine reference ranges for Simmental hematology, serum chemistry and enzymes activities. Values outside of three times interquartile range from the 25th and 75th percentile were defined as outliers and eliminated. Thereafter, the reference ranges were determined non-parametrically as interval between the 2.5th and 97.5th percentile.

Hematological and biochemical parameters of SA 4, SA 6, and SA 8 were compared with these reference ranges. Values of SA 20 and SA 22 were compared with those of $B$. besnoiti seronegative Limousin cattle (see below).

\section{Herd-BbGER1: monitoring of B. besnoiti seropositive Limousin cattle}

In total, 75 samples were collected from 54 female Limousin cattle (one to eleven years old) during routine herd health status examinations in the years 2008, 2011, and 2012. The sampling of these animals was conducted according to international guidelines and national law concerning animal welfare. Limousin cattle were free of BHV-1 and BVD infection and did not show clinical signs of gastrointestinal helminthoses or lung worm disease. All animals were seropositive for B. besnoiti antibodies in immunoblots and IFAT [24] and 32 animals displayed cysts in the scleral conjunctivae and/or vaginal vestibula. Only single animals showed large numbers of parasitic cysts in the vaginal vestibula and the scleral conjunctivae as well as visible and palpable lesions in the skin of the trunk, limbs and udder.

Values outside of three times interquartile range from the 25th and 75th percentile were defined as outliers and eliminated. After that, the 2.5th and 97.5th percentiles were determined non-parametrically.

\section{Herd-BbGER 1: monitoring of B. besnoiti negative Limousin cattle}

Samples were collected from two groups of female adult cattle of Herd-BbGER1. Fifteen samples from 14 healthy female Limousin cattle (one to eight years old) were collected during routine herd health status examinations in 2008, 2011 and 2012. The sampling of these animals was conducted according to international guidelines and national law concerning animal welfare.

Twenty-seven Limousin cows (two to 14 years old) without a history of bovine besnoitiosis were introduced into Herd-BbGER1 in spring 2013. Samples from these animals were collected as part of the quarantine health check. Limousin cattle from both groups were free of BHV-1 and BVD infection and did not show clinical signs of gastrointestinal helminthoses or lung worm disease and sera were negative for $B$. besnoiti antibodies in immunoblots and IFAT as previously described [24].

To determine Limousin reference ranges, values outside of three times interquartile range from the 25th and 75th percentile were defined as outliers and eliminated. After that, reference ranges were determined nonparametrically as interval between the 2.5 th and 97.5th percentiles.

\section{Blood sample collection and processing}

Blood samples were collected from the tail or jugular veins. Immediately after sampling, a blood smear was performed on a glass slide for differentiation count of cells. All blood samples were stored at approximately $8^{\circ} \mathrm{C}$ for up to 6 hours until transferred to the laboratory. Sera for antibody detection and serum protein electrophoresis were frozen at $-80^{\circ} \mathrm{C}$ until transferred to the Friedrich-LoefflerInstitut (Wusterhausen, Germany) and Vet Med Labor $\mathrm{GmbH}$ (Division of IDEXX Laboratories, Ludwigsburg, Germany) for further examination. Samples for serum chemistry, hematology and enzyme activities were kept overnight at $8^{\circ} \mathrm{C}$ and were processed the next day.

\section{Biochemical analysis and hematological examination}

Urea, creatinine, total protein (TP), albumin, total bilirubin, conjugated bilirubin, calcium, magnesium, phosphor, sodium, chloride and potassium concentrations, aspartate transaminase (AST), glutamate dehydrogenase (GLDH), gamma-glutamyl transpeptidase (GGT), creatine kinase (CK) activities were analyzed in sera with a Hitachi 912 Chemistry Analyzer (Boehringer Mannheim, Mannheim, Germany) in the laboratory of the Clinic for Ruminants. Globulin, albumin/globulin (A/G) ratio and unconjugated bilirubin were determined arithmetically.

A complete blood cell count was performed using EDTA-anticoagulated blood, analyzed with a hematological analyzer (pocH-100iV DIFF, Sysmex, Norderstedt, Germany). Air-dried slides for differential blood count were stained using a Pappenheim stain (Haema Schnellfaerbung, LT-SYS $^{\oplus}$ Labor + Technik Eberhard Lehmann GmbH, Berlin, Germany). Mean corpuscular hemoglobin concentration $(\mathrm{MCHC})$, mean corpuscular volume $(\mathrm{MCV})$ and mean corpuscular hemoglobin $(\mathrm{MCH})$ were determined arithmetically. 


\section{Serum protein gel electrophoresis}

Sera from SA 4, SA 6, and SA 20 were examined on the day of seroconversion, 2 days post seroconversion $(d p s)$, $5 d p s$ and on day 225. Sera from SA 8 and SA 22 were examined likewise with the exception of serum from 2 dps. In addition, three sera from SA 4 and two sera from SA 6 were analyzed during the febrile phase before seroconversion. Serum protein gel electrophoresis was performed at Vet Med Labor GmbH (Division of IDEXX Laboratories, Ludwigsburg, Germany) with a semiautomated agarose gel electrophoresis system (HYDRA$\mathrm{SIS}^{\circ} 2$, Sebia, Norcross, USA).

Reference ranges were calculated from the 24 samples of SA 1 to SA 12 from the beginning and the mid of the trial as described above. In order to exclude possible effects of parasite circulation on the determined parameters in SA 4, SA 6, and SA 8, only samples taken before the calculated incubation period of 13 days [6] were used.

\section{Serological examinations}

To detect B. besnoiti antibodies in serum, three tests were performed as previously described: one IFAT and two immunoblots either based on B. besnoiti tachyzoite or bradyzoite antigen. A reciprocal positive cut-off titer of 200 was used in IFAT and recognition of at least four of ten bands in both immunoblots was regarded specific [24]. Animals were regarded as positive if two of the three serological tests yielded a positive result.

\section{Statistical analysis}

Hematological results, serum chemistry and enzyme activities of B. besnoiti seronegative and B. besnoiti seropositive Limousin cattle were compared statistically. Additionally, the same parameters of $B$. besnoiti seropositive subclinically affected and $B$. besnoiti seropositive clinically affected animals were compared statistically. To assess normality, D'Agostino and Pearson omnibus normality test was applied. To compare the two groups, the non-parametric Mann-Whitney-Test was performed. $P$ values below 0.01 where considered significant. Data were analyzed using the software Graphpad Prism 5.04 for Windows.

\section{Results}

Chronology of hematologic alterations during acute, subacute, and chronic bovine besnoitiosis

Shortly before seroconversion, infected animals SA 4, SA 6 , and SA 8 showed a period of decline in white blood cell (WBC) concentration followed by an increase. SA 20 and SA 22 showed only a consistent increase in WBC concentrations after seroconversion. In SA 4 and SA 6, the decline was below the Simmental reference range (Table 2) for four days shortly after entering the acute
Table 2 Mean, SD and percentiles for laboratory parameters of $B$. besnoiti negative Simmental cattle (9 animals, 224 samples)

\begin{tabular}{|c|c|c|c|}
\hline \multirow[t]{2}{*}{ Hematology } & \multirow[t]{2}{*}{ Mean \pm SD } & \multicolumn{2}{|c|}{ Percentiles } \\
\hline & & 2.5th & 97.5th \\
\hline $\mathrm{RBCs}(\mathrm{T} / \mathrm{l})$ & $7.45 \pm 0.8$ & 6.28 & 9.29 \\
\hline Hemoglobin (mmol/l) & $6.5 \pm 0.5$ & 5.6 & 7.6 \\
\hline HCT (\%) & $32.6 \pm 2.8$ & 27.9 & 38.3 \\
\hline MCV (fl) & $43.8 \pm 3.1$ & 36.1 & 48.9 \\
\hline $\mathrm{MCH}$ (fmol) & $0.9 \pm 0.1$ & 0.7 & 1.0 \\
\hline $\mathrm{MCHC}(\mathrm{mmol} / \mathrm{l})$ & $20.0 \pm 0.7$ & 18.6 & 21.6 \\
\hline WBCs (G/l) & $7.90 \pm 1.7$ & 3.80 & 10.70 \\
\hline \multicolumn{4}{|l|}{ Serum chemistry } \\
\hline Total protein (g/l) & $75.2 \pm 4.7$ & 66.3 & 83.0 \\
\hline Albumin (g/l) & $34.8 \pm 2.5$ & 30.5 & 39.2 \\
\hline Globulin (g/l) & $40.4 \pm 5.2$ & 29.2 & 49.3 \\
\hline A/G ratio & $0.86 \pm 0.16$ & 0.64 & 1.24 \\
\hline Urea (mmol/l) & $5.2 \pm 0.9$ & 3.5 & 6.8 \\
\hline Creatinine $(\mu \mathrm{mol} / \mathrm{l})$ & $97.5 \pm 16.5$ & 70.4 & 128.2 \\
\hline Total bilirubin $(\mu \mathrm{mol} / \mathrm{l})$ & $1.4 \pm 0.7$ & 0.1 & 2.8 \\
\hline Sodium (mmol/l) & $142.3 \pm 2.6$ & 137.0 & 147.0 \\
\hline Potassium (mmol/l) & $4.2 \pm 0.4$ & 3.4 & 5.0 \\
\hline Calcium (mmol/l) & $2.4 \pm 0.1$ & 2.3 & 2.7 \\
\hline Phosphorus (mmol/l) & $2.4 \pm 0.3$ & 1.8 & 3.1 \\
\hline Magnesium (mmol/l) & $0.9 \pm 0.1$ & 0.8 & 1.1 \\
\hline Chloride (mmol/l) & $100.8 \pm 2.6$ & 96.0 & 106.0 \\
\hline \multicolumn{4}{|l|}{ Enzymes } \\
\hline AST (IU/I) & $70.4 \pm 9.7$ & 50.6 & 89.4 \\
\hline GGT (IU/I) & $18.9 \pm 4.8$ & 11.0 & 27.8 \\
\hline GLDH (IU/I) & $12.5 \pm 7.4$ & 4.2 & 31.5 \\
\hline CK (IU/I) & $217.0 \pm 65.0$ & 133.2 & 377.0 \\
\hline
\end{tabular}

$\mathrm{SD}=$ standard deviation

stage (Figure 1A). Reduced WBC concentrations consisted of an equal reduction in the concentrations of neutrophils and lymphocytes.

A decline in red blood cell (RBC) concentration was observed in SA 4 lasting for 20 days (Figure 1B). RBC concentrations of SA 20 fluctuated below the lower limit of the Limousin reference range (Table 3) for the whole trial period.

SA 4 and SA 6 showed a decline in hemoglobin concentration and HCT shortly before seroconversion. SA 20 showed strong fluctuation below or around the lower limit of the reference range for the whole trial period (Figure $1 \mathrm{C}$ and D).

Mean corpuscular hemoglobin concentration (MCHC) showed strong fluctuations above the lower limit of reference range in all infected animals (data not shown). Mean corpuscular volume (MCV) and mean corpuscular 


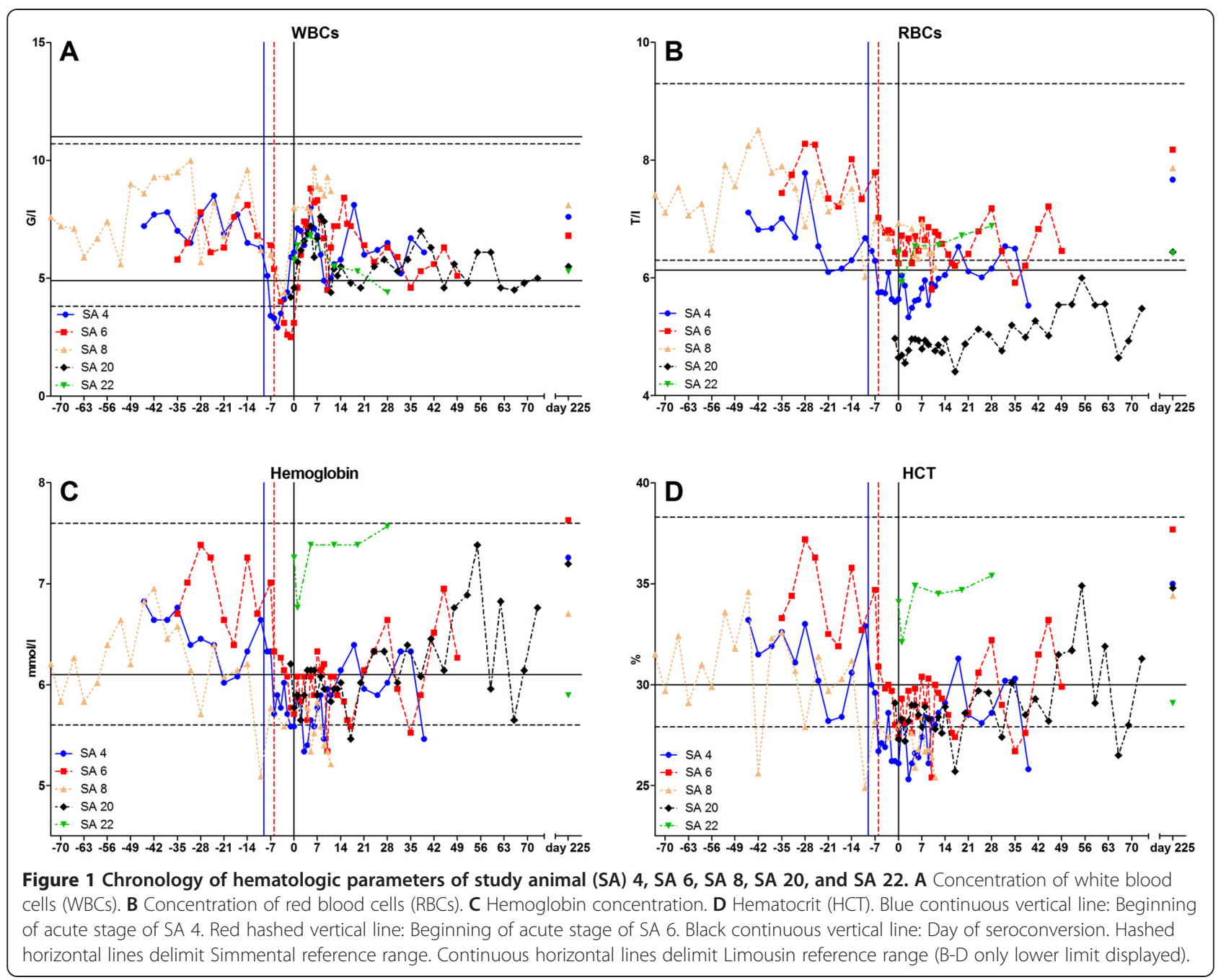

hemoglobin $(\mathrm{MCH})$ concentrations were within the reference range in all infected animals with few borderline values (data not shown).

\section{Chronology of changes in serum chemistry during acute,} subacute, and chronic bovine besnoitiosis

Elevations of TP and globulin concentrations plus decline in $\mathrm{A} / \mathrm{G}$ ratio were detectable shortly after seroconversion in SA 4, SA 6, SA 20, and SA 22 (Figure 2A and B).

Albumin concentration declined within reference range shortly after the beginning of the acute stage in SA 4. SA 20 displayed fluctuating albumin concentrations around the lower limit of the reference range during the whole trial period. SA 20 and SA 22 showed increasing albumin concentrations for few days after seroconversion.

On day 225 , albumin concentrations were within reference range in SA 20 and SA 22 and borderline in SA 4, SA 6, and SA 8.
Total bilirubin concentrations were above the reference range in SA 4, SA 20, and SA 22 (Figure 2C). In SA 20 and SA 22, this elevation was mainly caused by an elevated unconjugated bilirubin concentration (data not shown).

Urea concentrations were below the reference range for five days in SA 4 starting 4 days ante seroconversion (das) and for four days in SA 6 starting $4 d p s$. Lowest values were $2.8 \mathrm{mmol} / \mathrm{l}$ for SA 4 and $3.1 \mathrm{mmol} / \mathrm{l}$ for SA 6. SA 20 displayed an increased urea concentration on 1 das $(7.9 \mathrm{mmol} / \mathrm{l})$. SA 22 showed decreased urea concentration $(2.4 \mathrm{mmol} / \mathrm{l})$ for one day 5 dps (data not shown).

Creatinine concentrations were above the reference range in SA 4 for five days starting 8 das $(147 \mu \mathrm{mol} / \mathrm{l})$ and for three days starting 3 das in SA $6(135 \mu \mathrm{mol} / \mathrm{l})$. On day 225, total bilirubin, urea and creatinine concentrations were within the respective reference ranges in all animals except creatinine concentration in SA 22 $(100.8 \mu \mathrm{mol} / \mathrm{l})$ (data not shown).

Concentrations of magnesium (except SA 4, see below), phosphate, potassium, sodium and chloride displayed 
Table 3 Mean, SD and percentiles of laboratory parameters of $B$. besnoiti seronegative and $B$. besnoiti seropositive Limousin cattle

\begin{tabular}{|c|c|c|c|c|c|c|}
\hline \multirow{3}{*}{ Hematology } & \multicolumn{3}{|l|}{ Seronegative } & \multicolumn{3}{|l|}{ Seropositive } \\
\hline & \multirow[t]{2}{*}{ Mean \pm SD } & \multicolumn{2}{|c|}{ Percentiles } & \multirow[t]{2}{*}{ Mean \pm SD } & \multicolumn{2}{|c|}{ Percentiles } \\
\hline & & 2.5th & 97.5th & & 2.5th & 97.5th \\
\hline $\mathrm{RBCs}(\mathrm{T} / \mathrm{l})$ & $8.44 \pm 1.9^{a}$ & 6.13 & 13.70 & $7.08 \pm 1.2^{\mathrm{a}, \mathrm{b}}$ & 5.08 & 9.76 \\
\hline Hemoglobin $(\mathrm{mmol} / \mathrm{l})$ & $7.5 \pm 0.7$ & 6.1 & 8.9 & $7.9 \pm 1.3^{\mathrm{a}}$ & 5.8 & 10.7 \\
\hline HCT (\%) & $35.5 \pm 3.5$ & 30.0 & 41.3 & $36.3 \pm 5.6^{a}$ & 23.0 & 45.4 \\
\hline MCV (fl) & $43.9 \pm 9.4$ & 25.9 & 58.1 & $52.2 \pm 3.9^{b}$ & 43.9 & 58.8 \\
\hline $\mathrm{MCH}(\mathrm{fmol})$ & $0.9 \pm 0.2$ & 0.5 & 1.3 & $1.1 \pm 0.1^{b}$ & 0.9 & 1.3 \\
\hline $\mathrm{MCHC}(\mathrm{mmol} / \mathrm{l})$ & $21.2 \pm 0.9$ & 19.8 & 23.2 & $21.5 \pm 1.4^{\mathrm{a}}$ & 19.2 & 24.6 \\
\hline WBCs (G/l) & $7.34 \pm 1.6$ & 4.90 & 11.00 & $7.31 \pm 2.94^{\mathrm{a}}$ & 2.83 & 14.44 \\
\hline \multicolumn{7}{|l|}{ Serum chemistry } \\
\hline Total protein (g/l) & $68.4 \pm 5.9$ & 60.1 & 81.7 & $76.0 \pm 6.9^{b}$ & 63.1 & 89.1 \\
\hline Albumin (g/l) & $32.9 \pm 4.4$ & 26.0 & 41.2 & $36.4 \pm 2.7^{b}$ & 31.5 & 41.3 \\
\hline Globulin (g/l) & $35.3 \pm 4.3$ & 28.7 & 43.8 & $40.3 \pm 7.0^{\mathrm{a}, \mathrm{b}}$ & 28.5 & 55.5 \\
\hline A/G ratio & $0.95 \pm 0.18^{a}$ & 0.73 & 1.39 & $0.94 \pm 0.19^{a}$ & 0.63 & 1.38 \\
\hline Urea $(\mathrm{mmol} / \mathrm{l})$ & $3.9 \pm 1.3^{\mathrm{a}}$ & 2.6 & 7.0 & $5.2 \pm 1.9^{\mathrm{a}, \mathrm{b}}$ & 2.2 & 9.6 \\
\hline Creatinine $(\mu \mathrm{mol} / \mathrm{l})$ & $163.6 \pm 21.2$ & 124.7 & 207.7 & $164.4 \pm 27.1^{\mathrm{a}}$ & 125.4 & 214.6 \\
\hline Total bilirubin $(\mu \mathrm{mol} / \mathrm{l})$ & $2.6 \pm 0.9$ & 1.0 & 4.4 & $2.9 \pm 1.2^{\mathrm{a}}$ & 0.9 & 5.5 \\
\hline Sodium (mmol/l) & $145.8 \pm 3.4$ & 139.0 & 153.0 & $141.1 \pm 3.1^{b}$ & 135.5 & 146.5 \\
\hline Potassium (mmol/l) & $5.3 \pm 0.9$ & 3.6 & 6.7 & $4.8 \pm 0.7^{b}$ & 3.4 & 6.0 \\
\hline Calcium (mmol/l) & $2.3 \pm 0.1$ & 2.1 & 2.6 & $2.3 \pm 0.2$ & 1.9 & 2.6 \\
\hline Phosphorus (mmol/l) & $1.6 \pm 0.3$ & 1.1 & 2.3 & $1.9 \pm 0.4^{b}$ & 1.3 & 2.8 \\
\hline Magnesium (mmol/l) & $0.6 \pm 0.2$ & 0.3 & 1.1 & $0.9 \pm 0.1^{a, b}$ & 0.7 & 1.1 \\
\hline Chloride (mmol/l) & $99.2 \pm 2.3$ & 95.6 & 103.2 & $96.7 \pm 2.6^{b}$ & 91.6 & 101.5 \\
\hline \multicolumn{7}{|l|}{ Enzymes } \\
\hline AST (IU/I) & $155.1 \pm 61.9^{\mathrm{a}}$ & 68.9 & 323.4 & $102.2 \pm 31.6^{\mathrm{a}, \mathrm{b}}$ & 58.2 & 166.0 \\
\hline GGT (IU/I) & $16.6 \pm 6.6$ & 6.8 & 28.7 & $19.5 \pm 9.1$ & 1.7 & 35.4 \\
\hline GLDH (IU/I) & $14.7 \pm 7.1^{\mathrm{a}}$ & 5.2 & 29.6 & $12.7 \pm 8.1^{\mathrm{a}}$ & 4.0 & 33.2 \\
\hline CK (IU/I) & $702.8 \pm 466.8^{a}$ & 78.0 & 2092.0 & $331.9 \pm 283.1^{a, b}$ & 106.5 & 885.0 \\
\hline
\end{tabular}

Samples from $B$. besnoiti seronegative Limousins comprise 41 samples from 41 animals, samples from $B$. besnoiti seropositive Limousins comprise 75 samples

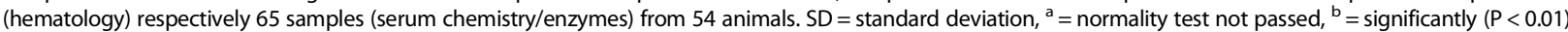
different versus seronegative Limousins (Mann-Whitney-Test) highlighted in bold.

strong fluctuations within the respective reference ranges in all infected animals, with single values lying outside the upper or lower limits (data not shown). SA 4 displayed a decline of magnesium concentrations starting 6 das for four days below reference range. Calcium concentrations declined in all three Simmentals shortly before seroconversion and were below the reference range in SA 4 and SA 20 (Figure 2D).

\section{Chronology of serum protein gel electrophoresis during acute, subacute, and chronic bovine besnoitiosis}

During the acute stage, SA 4 displayed no alterations of serum protein fractions compared with the Simmental reference range (Table 4). SA 6 displayed a slightly elevated $\alpha_{1}$-globulin-fraction $(7.1 \mathrm{~g} / \mathrm{l})$ and a diminished $\alpha_{2}$ - globulin-fraction $(2.4 \mathrm{~g} / \mathrm{l}) 7$ das. Alterations of serum protein fractions on the day of seroconversion and thereafter are depicted in Figure 3A-D.

\section{Chronology of enzyme activities during acute, subacute,} and chronic bovine besnoitiosis

AST activities are depicted in Figure 4.

CK activities were elevated for one day $3 \mathrm{dps}$ in SA 4 $(1,115 \mathrm{U} / \mathrm{l})$ and 1 das in SA 20 (2,900 U/l). Serum activities of GGT and GLDH remained below the upper limit of the reference range in all infected animals.

On day 225, activities of GGT, GLDH, and CK remained below the upper limit of the reference range in all animals. 


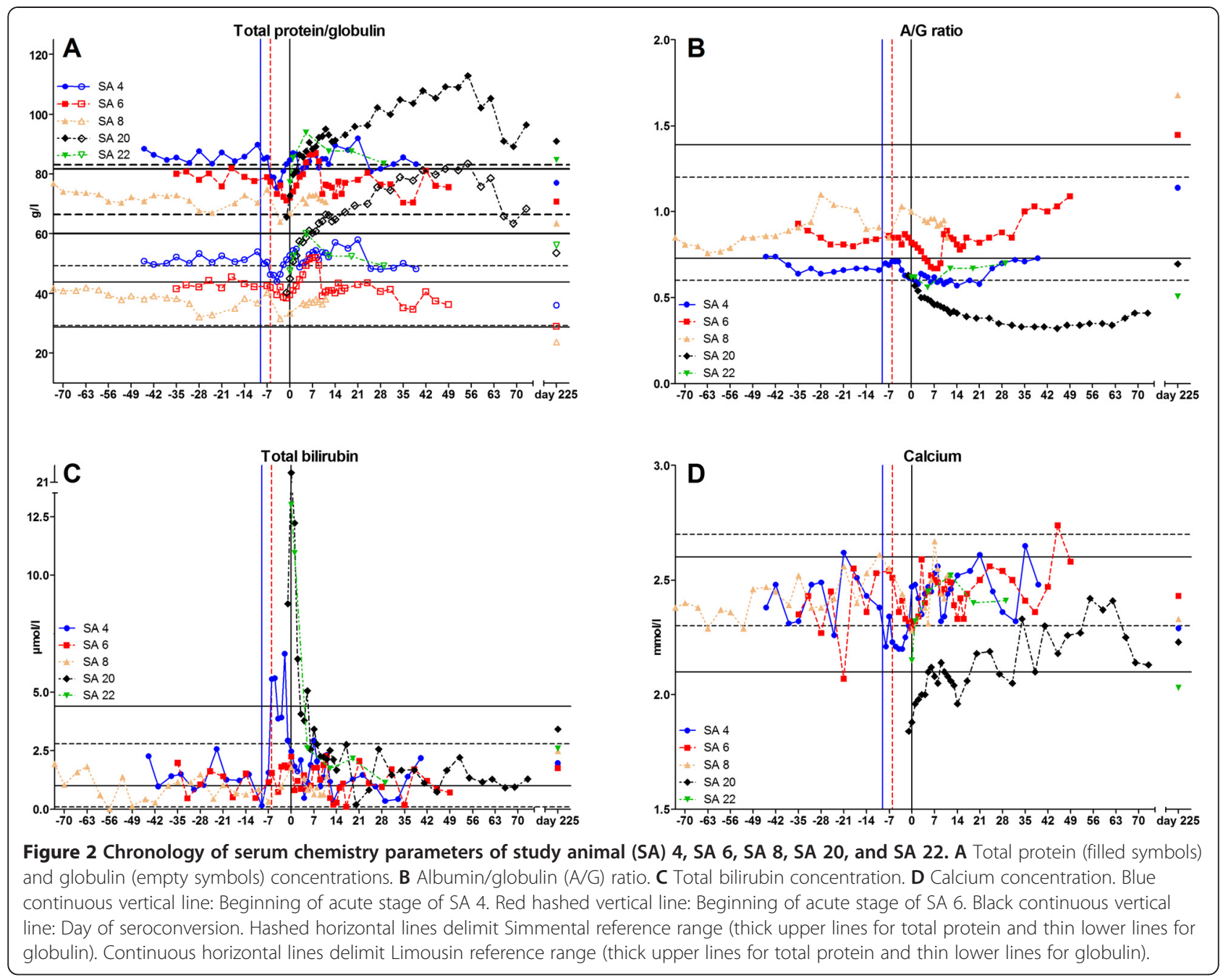

Herd-BbGER1 monitoring: significant differences in laboratory parameters between $B$. besnoiti seronegative and $B$. besnoiti seropositive Limousin cattle

Means, standard deviations and percentiles of hematology, serum chemistry and enzyme activities for $B$. besnoiti seronegative and $B$. besnoiti seropositive Limousin cattle are shown in Table 3.

The RBC concentration was significantly higher and $\mathrm{MCV}$ and $\mathrm{MCH}$ were significantly lower in B. besnoiti seronegative Limousins. TP, albumin and globulin concentrations were significantly higher in $B$. besnoiti seropositive Limousins.

AST and CK activities were significantly lower in $B$. besnoiti seronegative Limousins (Table 3).

There were no statistical significant differences between parameters of $B$. besnoiti seropositive subclinically affected and B. besnoiti seropositive clinically affected Limousin cattle.

During chronic besnoitiosis, laboratory parameters of SA 20 and SA 22 were within the percentiles of the $B$. besnoiti seropositive Limousin group, except RBC and albumin concentrations which were lower in SA 20 and globulin concentration which was higher in SA 20.

\section{Discussion}

The results of this study present the chronology of laboratory parameters during a longitudinal clinical trial monitoring five cattle with naturally acquired acute, subacute, and chronic bovine besnoitiosis. In addition, the laboratory parameters of $B$. besnoiti seronegative and $B$. besnoiti seropositive chronically infected Limousin cattle from a herd naturally affected by bovine besnoitiosis are compared.

Variations exist between reference ranges of different laboratories, cattle breeds, age groups, lactation and pregnancy status [26-31]. Thus, reference ranges for the evaluation of laboratory parameters of $B$. besnoiti infected Simmental cattle were calculated from values of healthy $B$. besnoiti negative Simmental study animals. Although larger animal cohorts are usually used for 
Table 4 Mean, SD and percentiles of serum gel electrophoresis of $B$. besnoiti negative Simmental cattle (12 animals, 24 samples) and comparison with results from the *2-year-group (24 animals) of Alberghina et al. [25]

\begin{tabular}{|c|c|c|c|c|}
\hline \multicolumn{4}{|c|}{ Data obtained in the present study } & \multirow{3}{*}{$\begin{array}{l}\text { Literature data* } \\
\text { Mean } \pm \text { SD }\end{array}$} \\
\hline \multirow{2}{*}{$\begin{array}{l}\text { Serum Gel } \\
\text { Electrophoresis }\end{array}$} & \multirow[t]{2}{*}{ Mean \pm SD } & \multicolumn{2}{|c|}{ Percentiles } & \\
\hline & & 2.5th & 97.5th & \\
\hline Total protein $(\mathrm{g} / \mathrm{l})$ & $64.92 \pm 3.55$ & 59.58 & 70.85 & $68.10 \pm 10.13$ \\
\hline Albumin (\%) & $49.4 \pm 4.6$ & 40.3 & 56.1 & Not given \\
\hline a1-globulins (\%) & $5.7 \pm 0.8$ & 4.5 & 7.3 & Not given \\
\hline a2-globulins (\%) & $9.4 \pm 1.4$ & 6.5 & 11.2 & Not given \\
\hline$\beta$-globulins (\%) & $13.2 \pm 2.1$ & 8.2 & 16.7 & Not given \\
\hline Y-globulins (\%) & $22.4 \pm 6.0$ & 14.3 & 36.1 & Not given \\
\hline Albumin (g/l) & $32.04 \pm 3.35$ & 25.45 & 36.85 & $31.79 \pm 5.12$ \\
\hline a1-globulins (g/l) & $3.67 \pm 0.42$ & 3.06 & 4.54 & $5.92 \pm 2.54$ \\
\hline a2-globulins (g/l) & $5.96 \pm 0.68$ & 4.68 & 7.19 & $5.77 \pm 1.73$ \\
\hline$\beta$-globulins (g/l) & $8.54 \pm 1.47$ & 5.58 & 11.43 & $7.50 \pm 1.16$ \\
\hline Y-globulins (g/l) & $14.55 \pm 4.22$ & 9.45 & 23.85 & $16.81 \pm 3.72$ \\
\hline
\end{tabular}

$\mathrm{SD}=$ standard deviation

calculation, these reference ranges were regarded representative for this study, because housing, feeding and handling were similar and examination of cattle samples of the same breed were examined in the same laboratories. For the same reason, laboratory parameters of SA 20 and SA 22 were compared with reference ranges calculated from healthy $B$. besnoiti seronegative female Limousin cattle of Herd-BbGER1. Furthermore, we could not find appropriate up-to-date reference values for adult Limousin cows in the literature and application of older data could have caused misinterpretations because reference values may change due to genetic and environmental factors [32].

\section{The effect of bovine besnoitiosis on hematological parameters}

Several hematological parameters were altered during acute bovine besnoitiosis. In the acute stage, leukopenia was observed in SA 4, SA 6, and SA 20. Leukopenia is a common finding in cattle with viral, bacterial and protozoan infections $[33,34]$ and is due to the low storage neutrophil pool of adult cattle as well as increased margination and tissue emigration during acute inflammation.

For the low RBC concentrations in SA 4 and SA 20 there are two possible explanations: First, multifocal hemorrhages, which have been described in acute besnoitiosis [15] and were also clinically observed in SA 4 [23] and in multiple consecutive histological skin sections taken during acute besnoitiosis in these animals [35]. However, total bilirubin concentrations - although of limited value as a confirmation of hemorrhages in sick cattle (see below) - returned to the normal range after a few days, indicating that hemorrhages are not the only reason contributing to this finding. Second, anemia can also be a result of a chronic inflammatory disease $[36,37]$. The constantly low RBC concentrations of SA 20 and the lower $\mathrm{RBC}$ concentrations in the seropositive Limousin group are most likely caused by the chronic inflammatory state. Reticulocyte stains of blood smears to assess the origin of anemia were not performed and reticulocytes were not observed in conventionally stained smears. Similar significant differences in hematological parameters, namely increased MCV and $\mathrm{MCH}$ and decreased RBC concentrations were also observed in Iranian goats infected by B. caprae [21].

\section{The effect of bovine besnoitiosis on serum chemistry values}

The initial hypothesis of increased globulin concentrations during chronic bovine besnoitiosis proved true. However, the effect of besnoitiosis on albumin concentrations was different than expected.

A decrease in albumin synthesis, albumin loss or hemodilution can be the cause of SA 20's hypoalbuminemia [38]. However, clinical examinations and the results of laboratory tests did not reveal evidence for albumin loss or hemodilution. Two effects probably caused the decreased albumin synthesis in SA 20: inflammation and a negative energy balance. Albumin is a negative acute phase protein and inflammatory hypoalbuminemia can develop after ongoing inflammatory states and is usually expected to be mild [38]. Moreover, it is tempting to speculate that the massive development of numerous $B$. besnoiti cysts in the tissues of SA 20 led to an increased demand in nutrients and subsequently to a negative energy balance. The hypocalcemia in this animal is very likely associated with hypoalbuminemia [39], as clinical signs of hypocalcemia were not observed.

The increased globulin concentrations are most likely due to increased antibodies against $B$. besnoiti, since reciprocal IFAT titers displayed a similar increase post seroconversion [23]. This hypothesis is supported by the electrophoresis findings displaying increased $\gamma$-globulin fractions $5 d p s$ in SA 20 and on day 225 in SA 20 and SA 22.

The increase in the $\alpha_{1}$-fraction ( $\alpha_{1}$-lipoprotein, $\alpha_{1}$ antitrypsin, and $\alpha_{1}$-antichymotrypsin) during the febrile phase of SA 6 and the increase in both $\alpha_{1}$ - and $\alpha_{2}$-fractions $\left(\alpha_{2}\right.$-macroglobulin and haptoglobins) of SA 4, SA 6, SA 20, and SA 22 shortly after seroconversion are most likely a response to the acute inflammation. Interestingly, the $\alpha$-fraction was only elevated during the febrile phase in SA 6 on one day. A reason for this may be the rather mild clinical course of the disease in SA 4 and 


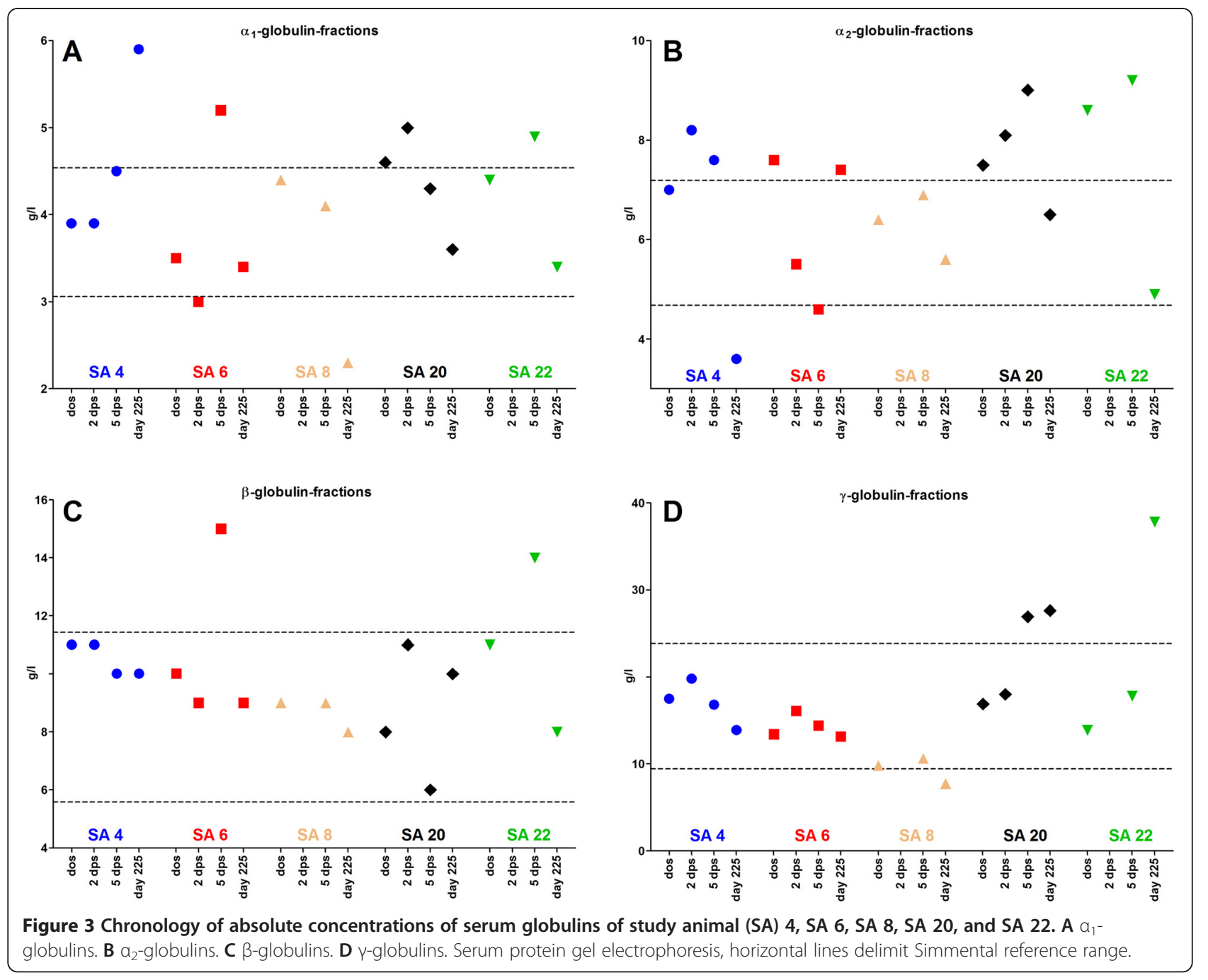

SA 6. The increase in the $\beta$-fraction of SA 6 and SA 22 on $5 \mathrm{dps}$ is most likely due to an increase in IgMantibodies or complement proteins, because transferrin, a main part of the $\beta$-fraction is expected to be low during acute inflammation [40]. SA 20 's elevated $\gamma$-globulin fraction (mainly IgG) displayed a two-peak pattern 5 $d p s$, and elevated $\gamma$-globulin fractions of the other days displayed only one peak. This is indicative of a polyclonal hypergammaglobulinemia shortly after seroconversion changing into a monoclonal or oligoclonal gammoglobulinemia in the chronic stage.

The significant hyperproteinemia of B. besnoiti seropositive Limousin cows of Herd-BbGER1 compared with $B$. besnoiti seronegative Limousin cows is mainly caused by hyperglobulinemia. These findings correlate with the results obtained from the trial animals. Elevation of $\gamma$-globulin concentration is common during chronic inflammatory diseases [38], and similar results have also been reported in goats suffering from natural chronic caprine besnoitiosis [20]. Unexpectedly, albumin concentrations were higher in $B$. besnoiti seropositive Limousins. Albumin tends to decrease during inflammatory states (see above), and decreased albumin concentrations have also been found in cases of caprine besnoitiosis [41]. Maybe the higher albumin concentrations are due to a slight dehydration of these animals, which is also indicated by slightly higher urea concentrations of $B$. besnoiti seropositive Limousins compared with $B$. besnoiti seronegative Limousins. Severely infected Limousins in this study had a tendency to have higher total protein and globulin concentrations as well as lower $\mathrm{A} / \mathrm{G}$ ratios. The former two observations were also made in goats naturally infected with B. caprae in Iran [20].

The hyperbilirubinemia of SA 4, SA 20, and SA 22 most likely has two main causes: A prehepatic icterus following hemorrhages (see above), or anorexia and sickness, which are common reasons for hyperbilirubinemia in different species (including cattle) and can include elevated unconjugated bilirubin concentrations [42-45]. 


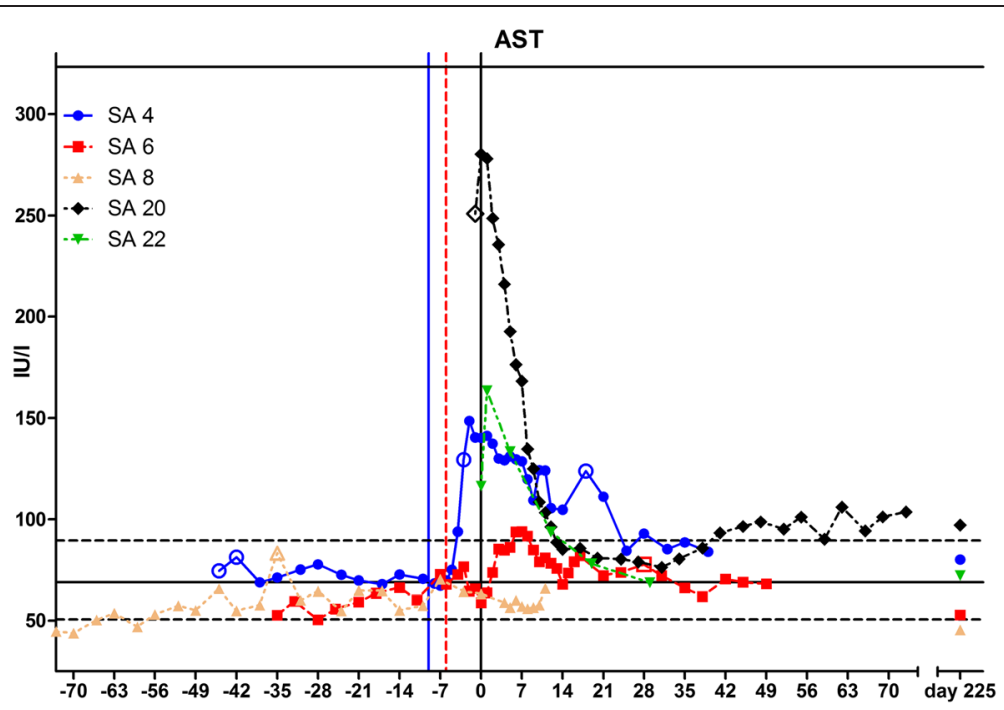

Figure 4 Chronology of aspartate transaminase (AST) activities of study animal (SA) 4, SA 6, SA 8, SA 20, and SA 22 . The empty symbols depict the days on which creatine kinase activities were above the respective reference range. Blue continuous vertical line: Beginning of acute stage of SA 4. Red hashed vertical line: Beginning of acute stage of SA 6. Black continuous vertical line: Day of seroconversion. Hashed horizontal lines delimit Simmental reference range. Continuous horizontal lines delimit Limousin reference range.

The decrease in urea concentrations in SA 4 and SA 6, as well as low and borderline magnesium concentrations observed for 7 das in SA 4 can be associated with a period of anorexia in these animals, too. The elevation of creatinine concentrations in SA 4 and SA 6 are most likely due to dehydration [46]. SA 4 and SA 6 showed clinical dehydration on several days and the dehydration period coincides with the anorectic period [23]. Urea/ creatinine ratio to further assess azotemia was not helpful in these cases, because urea concentrations were reduced.

Electrolyte concentrations in blood are influenced by feed intake, hydration status or sweating and are subject to daily change, as it was observed in the trial animals. These factors are also a plausible explanation for the occasional changes observed in the two Limousin groups. Using our data to conclude that bovine besnoitiosis affects electrolyte concentrations seems inappropriate at this point and further studies should be conducted to assess the importance of these alterations.

\section{The effect of bovine besnoitiosis on enzyme activities}

The increased AST activities of SA 4 and SA 20 are most likely associated with a loss of muscle fiber integrity, because CK activities were elevated at the same time and GLDH and GGT displayed no elevated activities. The longer half-life of AST $[47,48]$ explains the relatively rapid decline of CK activities and the slow removal of AST from serum. Muscle fiber damage may be caused by prolonged recumbence, because $B$. besnoiti affected cattle show apathy and are more likely to lie down and rest [49]. Or elevated AST activities could be a result of mild muscular degeneration and necrosis, lesions which are described in cattle during acute besnoitiosis after experimental infection with high parasite doses [15].

Although lower AST and CK activities are of little clinical significance, it is surprising that AST and CK activities were lower in $B$. besnoiti seropositive Limousins compared with $B$. besnoiti seronegative Limousins. Tissue cysts surrounded by severe chronic inflammatory reaction can be found in the musculature of diseased cattle [9], and loss of muscle fiber integrity due to cysts or inflammation seems likely. Maybe the effect of tissue cysts on muscle fiber integrity is too low to get noticed or leads to mild, gradual release of muscle enzymes, which cannot be detected due to the short half-life of CK. In comparison with the results obtained from the trial animals, loss of muscle fiber integrity seems to play only a role during acute and subacute besnoitiosis.

\section{Conclusions}

This study provides detailed results of laboratory parameters obtained during the course of naturally acquired acute, subacute and chronic bovine besnoitiosis for the first time. Even though a low number of animals were examined in acute and subacute besnoitiosis, it could be shown that hematologic parameters were altered especially during acute and subacute besnoitiosis, leading to reduced red and white blood cell concentrations. Furthermore, during acute and subacute besnoitiosis, elevation of serum globulin fractions reflected the acute inflammatory state, clinical parameters like disturbed condition resulted in increased bilirubin concentrations and lesions like muscle necroses, which are described in 
the literature led to increased aspartate transaminase and creatine kinase activities. Chronic besnoitiosis led to reduced concentrations of red blood cells and to hyper(gamma)-globulinemia most likely due to the chronic inflammatory condition caused by the parasite.

\section{Abbreviations}

A/G ratio: Albumin/globulin ratio; AST: Aspartate transaminase; CK: Creatine kinase; das: Days ante seroconversion; dps: Days post seroconversion; GGT: Gamma-glutamyl transpeptidase; GLDH: Glutamate dehydrogenase; HCT: Hematocrit; Herd-BbGER1: First German cattle herd diagnosed with cases of bovine besnoitiosis; IFAT: Immunofluorescent antibody test; MCH: Mean corpuscular hemoglobin; MCHC: Mean corpuscular hemoglobin concentration; MCV: Mean corpuscular volume; RBCs: Red blood cells; SA: Study animal; td: Trial day; WBCs: White blood cells.

\section{Competing interests}

The authors declared no potential conflicts of interest with respect to the research, authorship, and/or publication of this article. Prionics AG, Schlieren, Switzerland funded parts of the study but had no influence on the design of the experiment, data collection and analysis and was not involved in writing the manuscript.

\section{Authors' contributions}

MCL collected the samples, performed the data management and analysis and wrote the manuscript. JCS collected the samples and performed data management. CS did the statistical workup and revised the manuscript. GS designed and coordinated the serological investigations and performed the respective data analysis. NSG was involved in sample collection, data analysis, study design and helped drafting the manuscript. All authors read and approved the final manuscript.

\section{Acknowledgements}

We acknowledge the excellent technical assistance of Ingrid Hartmann, Christina Beyer, and Monika Altmann. We also like to express our gratitude to the owner of the animals for his collaboration and support. Partial financial support for this study was provided by Prionics AG (Schlieren, Switzerland) and Vet Med Labor GmbH (Division of IDEXX Laboratories, Ludwigsburg, Germany).

\section{Author details}

${ }^{1}$ Institute of Veterinary Pathology at the Centre for Clinical Veterinary Medicine, Ludwig-Maximilians-Universitaet Muenchen, Munich, Germany. ${ }^{2}$ Rammingen, Germany. ${ }^{3} \mathrm{Clinic}$ for Ruminants with Ambulatory and Herd Health Services at the Centre for Clinical Veterinary Medicine, Ludwig-Maximilians-Universitaet Muenchen, Oberschleissheim, Germany. ${ }^{4}$ Friedrich-Loeffler-Institut, Federal Research Institute for Animal Health, Institute of Epidemiology, Greifswald-Isle of Riems, Germany.

\section{Received: 14 May 2014 Accepted: 13 January 2015}

\section{Published online: 13 February 2015}

\section{References}

1. Hornok S, Fedak A, Baska F, Hofmann-Lehmann R, Basso W. Bovine besnoitiosis emerging in Central-Eastern Europe, Hungary. Parasit Vectors. 2014;7(1):20.

2. Lesser M, Braun U, Deplazes P, Gottstein B, Hilbe M, Basso W. [First cases of besnoitiosis in cattle in Switzerland]. Schweiz Arch Tierheilkd. 2012;154(11):469-74.

3. Gentile A, Militerno G, Schares G, Nanni A, Testoni S, Bassi P, et al. Evidence for bovine besnoitiosis being endemic in Italy - first in vitro isolation of Besnoitia besnoiti from cattle born in Italy. Vet Parasitol. 2012;184(2-4):108-15.

4. Rostaher A, Mueller RS, Majzoub M, Schares G, Gollnick NS. Bovine besnoitiosis in Germany. Vet Dermatol. 2010;21(4):329-39.

5. EFSA. European food safety authority - Bovine Besnoitiosis: an emerging disease in Europe. EFSA J. 2010;8(2):1499.

6. Bigalke RD. New concepts on the epidemiological features of bovine besnoitiosis as determined by laboratory and field investigations. Onderstepoort J Vet Res. 1968;35(1):3-137.

7. Pols JW. Studies on bovine besnoitiosis with special reference to the aetiology. Onderstepoort J Vet Res. 1960;28:265-356.
8. Schulz KCA. A report on naturally acquired besnoitiosis with special reference to its pathology. J S Afr Vet Med Assoc. 1960;31:21-35.

9. McCully RM, Basson PA, Van Niekerk JW, Bigalke RD. Observations on Besnoitia cysts in the cardiovascular system of some wild antilopes and domestic cattle. Onderstepoort J Vet Res. 1966;33:245-76.

10. Majzoub M, Breuer W, Gollnick NS, Rostaher A, Schares G, Hermanns W. Ein Ausbruch von Besnoitiose bei Rindern in Deutschland: pathomorphologische, ultrastrukturelle und molekularbiologische Untersuchungen. Wien Tieraerztl Mschr. 2010;97:9-15.

11. Franko EE, Borges I. Sur la sarcosporidiose bovine. Arq Inst Bac Camera Pestana. 1915;269-289

12. Garcia-Lunar $P$, Ortega-Mora LM, Schares G, Gollnick NS, Jacquiet $P$, Grisez C, et al. An inter-laboratory comparative study of serological tools employed in the diagnosis of Besnoitia besnoiti infection in bovines. Transbound Emerg Dis. 2012

13. Schares G, Langenmayer MC, Scharr JC, Minke L, Maksimov P, Maksimov A, et al. Novel tools for the diagnosis and differentiation of acute and chronic bovine besnoitiosis. Int J Parasitol. 2013:43(2):143-54.

14. Basso W, Schares G, Gollnick NS, Rutten M, Deplazes P. Exploring the life cycle of Besnoitia besnoiti - Experimental infection of putative definitive and intermediate host species. Vet Parasitol. 2011;178(3-4):223-34.

15. Basson PA, McCully RM, Bigalke RD. Observations on the pathogenesis of bovine and antelope strains of Besnoitia besnoiti (Marotel, 1912) infection in cattle and rabbits. Onderstepoort J Vet Res. 1970;37(2):105-26.

16. Dubey JP, Shkap V, Pipano E, Fish L, Fritz DL. Ultrastructure of Besnoitia besnoiti tissue cysts and bradyzoites. J Eukaryot Microbiol. 2003;50(4):240-4.

17. Ellis JT, Holmdahl OJ, Ryce C, Njenga JM, Harper PA, Morrison DA. Molecular phylogeny of Besnoitia and the genetic relationships among Besnoitia of cattle, wildebeest and goats. Protist. 2000;151(4):329-36.

18. Namazi F, Oryan A, Sharifiyazdi H. Genetic characterization of the causative agent of besnoitiosis in goats in Iran on the basis of internal transcribed spacer rDNA and its comparison with Besnoitia species of other hosts. Parasitol Res. 2011;108(3):633-8.

19. Njenga JM, Bwangamoi O, Mutiga ER, Kangethe EK, Mugera GM. Preliminary findings from an experimental study of caprine besnoitiosis in Kenya. Vet Res Commun. 1993;17(3):203-8.

20. Oryan A, Nazifi S, Mohebbi H. Pathology and serum biochemical changes in natural caprine besnoitiosis. Rev Med Vet. 2008;159(1):27-32.

21. Nazifi S, Oryan A, Mohebbi M. Evaluation of Hematological Parameters in Caprine Besnoitiosis. J Appl Anim Res. 2002;21:123-8.

22. Besnoit C, Robin V. Sarcosporidiose cutanée chez une vache. Rev Vét. 1912;37:649-63.

23. Gollnick NS, Scharr JC, Schares G, Langenmayer MC. Natural Besnoitia besnoiti infections in cattle: chronology of disease progression. BMC Vet Res. accepted 2015.

24. Schares G, Basso W, Majzoub M, Rostaher A, Scharr J-C, Langenmayer MC, et al. Comparative evaluation of immunofluorescent antibody and new immunoblot test for the specific detection of antibodies against Besnoitia besnoiti tachyzoites and bradyzoites in bovine sera. Vet Parasitol. 2010;171(1-2):32-40.

25. Alberghina D, Giannetto C, Vazzana I, Ferrantelli V, Piccione G. Reference intervals for total protein concentration, serum protein fractions, and albumin/globulin ratios in clinically healthy dairy cows. J Vet Diagn Invest. 2011;23(1):111-4

26. Brun-Hansen $\mathrm{HC}$, Kampen $\mathrm{AH}$, Lund $\mathrm{A}$. Hematologic values in calves during the first 6 months of life. Vet Clin Pathol. 2006;35(2):182-7.

27. Doornenbal H. Physiological and endrocine parameters in beef cattle: breed, sex and year differences. Can J Comp Med. 1977;41(1):13-8.

28. Doornenbal H, Tong AK, Murray NL. Reference values of blood parameters in beef cattle of different ages and stages of lactation. Can J Vet Res. 1988;52(1):99-105.

29. Monke DR, Kociba GJ, DeJarnette M, Anderson DE, Ayars Jr WH. Reference values for selected hematologic and biochemical variables in Holstein bulls of various ages. Am J Vet Res. 1998;59(11):1386-91.

30. Yokus B, Cakir UD. Seasonal and physiological variations in serum chemistry and mineral concentrations in cattle. Biol Trace Elem Res. 2006;109(3):255-66.

31. Knowles TG, Edwards JE, Bazeley KJ, Brown SN, Butterworth A, Warriss PD. Changes in the blood biochemical and haematological profile of neonatal calves with age. Vet Rec. 2000;147(21):593-8.

32. George JW, Snipes J, Lane VM. Comparison of bovine hematology reference intervals from 1957 to 2006. Vet Clin Pathol. 2010;39(2):138-48.

33. Andresen HA. Evaluation of leukopenia in cattle. J Am Vet Med Assoc. 1970;156(7):858-66. 
34. Glass EJ, Preston PM, Springbett A, Craigmile S, Kirvar E, Wilkie G, et al. Bos taurus and Bos indicus (Sahiwal) calves respond differently to infection with Theileria annulata and produce markedly different levels of acute phase proteins. Int J Parasitol. 2005;35(3):337-47.

35. Langenmayer MC, Gollnick NS, Majzoub-Altweck M, Scharr JC, Schares G, Hermanns W. Naturally acquired bovine besnoitiosis: histological and immunohistochemical findings in acute, subacute and chronic disease. Vet Pathol. 2014; doi: 101177/0300985814541705.

36. Stockham SL, Scott MA. Erythrocytes. In: Fundamentals of Veterinary Clinical Pathology. 2nd ed. Ames: Blackwell Publishing; 2008. p. 107-222.

37. Thrall MA. Classification of and diagnostic approach to anemia. In: Troy DB, editors. Veterinary Hematology and Clinical Chemistry. Lippincott Williams \& Wilkins; 2004. p. 83-8

38. Stockham SL, Scott MA. Proteins. In: Fundamentals of Veterinary Clinical Pathology. 2nd ed. Ames: Blackwell Publishing; 2008. p. 369-414.

39. Stockham SL, Scott MA. Calcium, phosphorus, magnesium, and their regulatory hormones. In: Fundamentals of Veterinary Clinical Pathology. 2nd ed. Ames: Blackwell Publishing; 2008. p. 593-638.

40. O'Connell TX, Horita TJ, Kasravi B. Understanding and interpreting serum protein electrophoresis. Am Fam Physician. 2005;71(1):105-12.

41. Nazifi S, Oryan A, Namazi F. Hematological and serum biochemical analyses in experimental caprine besnoitiosis. Korean J Parasitol. 2011;49(2):133-8.

42. Cakala S, Bieniek K. Bromosulphthalein clearance and total bilirubin level in cows deprived of food and water. Zentralbl Veterinaermed A. 1975;22(7):605-10.

43. Gronwall R, Engelking LR. Effect of glucose administration on equine fasting hyperbilirubinemia. Am J Vet Res. 1982;43(5):801-3.

44. Meyer BH, Scholtz HE, Schall R, Muller FO, Hundt HK, Maree JS. The effect of fasting on total serum bilirubin concentrations. Br J Clin Pharmacol. 1995;39(2):169-71.

45. McSherry BJ, Lumsden JH, Valli VE, Baird JD. Hyperbilirubinemia in sick cattle. Can J Comp Med. 1984;48(3):237-40.

46. Stockham SL, Scott MA. Urinary system. In: Fundamentals of veterinary clinical pathology. 2nd ed. Ames: Blackwell Publishing; 2008. p. 415-94.

47. Russell KE, Roussel AJ. Evaluation of the ruminant serum chemistry profile. Vet Clin North Am Food Anim Pract. 2007;23(3):403-26.

48. Lefebvre HP, Toutain PL, Serthelon JP, Lassourd V, Gardey L, Braun JP. Pharmacokinetic variables and bioavailability from muscle of creatine kinase in cattle. Am J Vet Res. 1994;55(4):487-93.

49. Bigalke RD. Besnoitiosis and Globidiosis. In: Ristic M, Mclntyre I, editors. Diseases of Cattle in Tropics. Volume 6, edn. The Hague: Martinus Nijhoff Publishers; 1981. p. 429-42.

\section{Submit your next manuscript to BioMed Central and take full advantage of:}

- Convenient online submission

- Thorough peer review

- No space constraints or color figure charges

- Immediate publication on acceptance

- Inclusion in PubMed, CAS, Scopus and Google Scholar

- Research which is freely available for redistribution 